\title{
scmap - A tool for unsupervised projection of single cell RNA-seq data
}

\author{
Vladimir Yu Kiselev ${ }^{1}$, Andrew Yiu ${ }^{1}$ and Martin Hemberg ${ }^{1}$ \\ ${ }^{1}$ Wellcome Trust Sanger Institute, Hinxton, Cambridge, UK
}

\section{Abstract}

Single-cell RNA-seq (scRNA-seq) is widely used to investigate the composition of complex tissues $^{1-9}$ since the technology allows researchers to define cell-types using unsupervised clustering of the transcriptome ${ }^{8,10}$. However, due to differences in experimental methods and computational analyses, it is often challenging to directly compare the cells identified in two different experiments. Here, we present scmap (http://bioconductor.org/packages/scmap), a method for projecting cells from a scRNA-seq experiment onto the cell-types or individual cells identified in other experiments (the application can be run for free, without restrictions, from http://www.hemberg-lab.cloud/scmap).

\section{Main text}

As more and more scRNA-seq datasets become available, carrying out comparisons between them is key. A central application is to compare datasets of similar biological origin collected by different labs to ensure that the annotation and the analysis is consistent. Moreover, as very large references, e.g. the Human Cell Atlas $(\mathrm{HCA})^{11}$, become available, an important application will be to project cells from a new sample (e.g. from a disease tissue) onto the reference to characterize differences in composition, or to detect new cell-types (Fig. 1a). Conceptually, such projections are similar to the popular BLAST ${ }^{12}$ method, which makes it possible to quickly find the closest match in a database for a newly identified nucleotide or amino acid sequence.

Projecting a new cell, $c$, onto a reference dataset, amounts to identifying which cluster or cell $c$ is most similar to, i.e. the nearest neighbor. We represent each cluster by its centroid, i.e. a vector of the median value of the expression of each gene, and we measure the similarity between $c$ and each cluster centroid or cell. Searching for the nearest cluster can be done exhaustively since the number of clusters is typically much smaller than the number of cells in the reference. To speed up the search for the nearest cell, we carry out an approximate nearest neighbor (ANN) search using a product quantizer ${ }^{13}$. Moreover, instead of using all genes when calculating the similarity, we use unsupervised feature selection to include only the genes that are most relevant for the underlying biological differences which allows us to overcome batch effects ${ }^{14}$.

We investigate three different strategies for feature selection: random selection, highly variable genes (HVGs) $)^{15}$ and genes with a higher number of dropouts than expected (M3Drop) ${ }^{14}$. To increase speed, we modified the M3Drop method and instead of fitting a Michaelis-Menten model 
to the log expression-dropout relation, we fit a linear model (Methods, Fig. S1a). For the number of features, we used the top 100, 200, 500, 1000, 2000, 5000, or all genes. Similarities were calculated using the cosine similarity, Pearson and Spearman correlations. This has the advantage of being insensitive to differences in scale between datasets as the similarities are restricted to the interval $[-1,1]$. To make the cluster assignments more robust, we required that at least two of the three similarities were in agreement, and that their value exceeded .7 for at least one of them. If these criteria are not met, then $c$ is labelled as "unassigned" to indicate that it does not correspond to any cell-type present in the reference. For the ANN search, which we refer to as scmap-cell, we carry out a form of k-nearest neighbour classification with only the cosine similarity. The nearest three neighbours are found and we require that they have the same cell-type and that the highest similarity among them to be $>.5$ for the cell-type to be assigned.

a

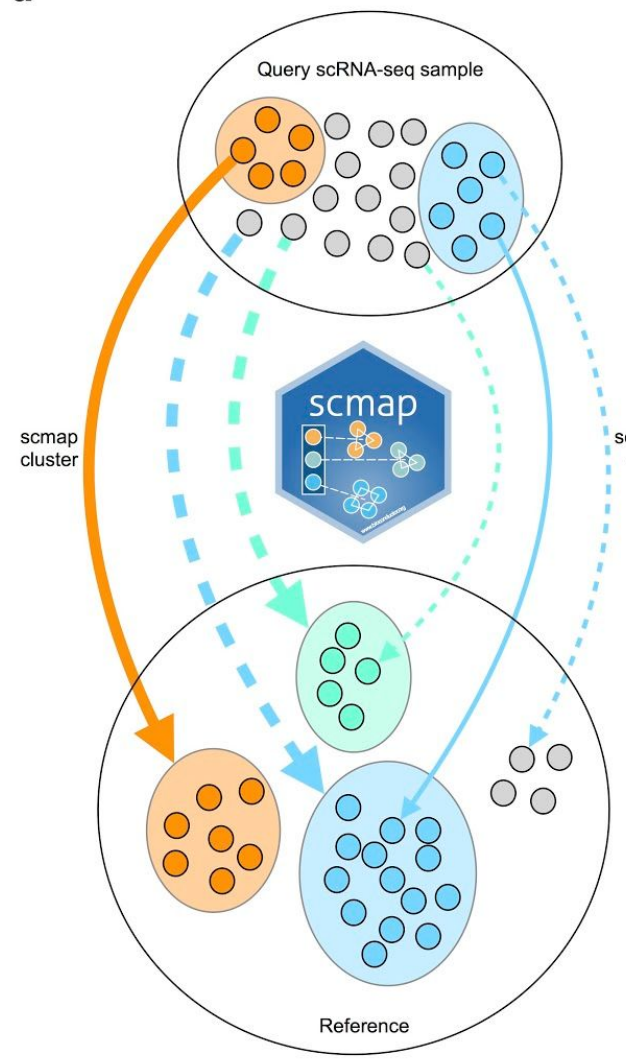

b

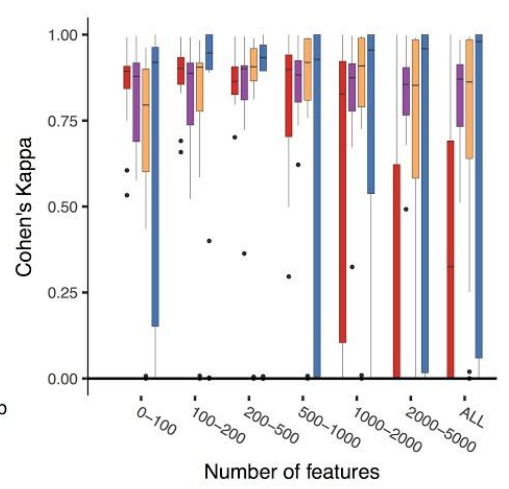

d

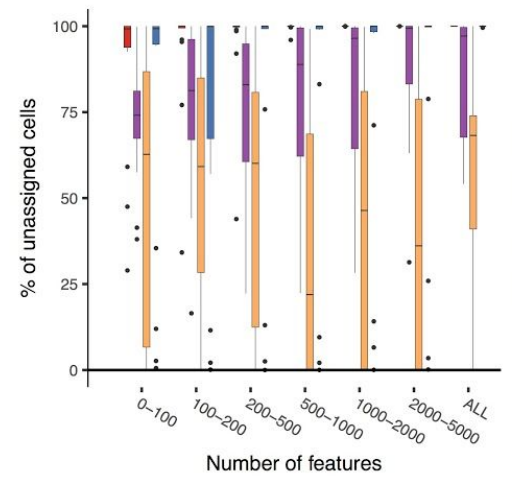

Method = scmap-cluster $=$ scmap-cell $\doteq \mathrm{SVM} \equiv \mathrm{RF}$

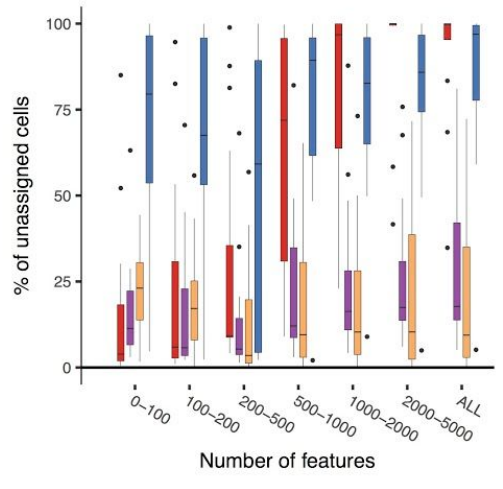

\# of NNs $=5$ = 10

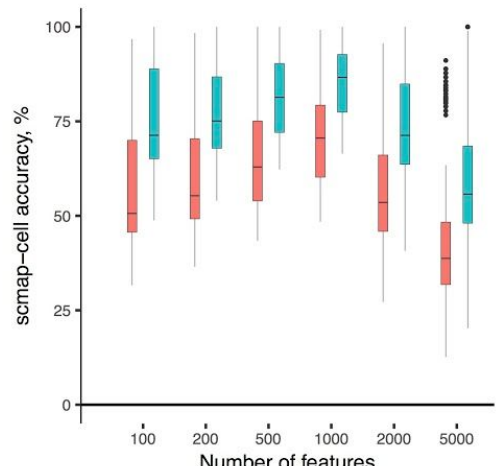

Figure 1 scmap use-cases and performance. (a) scmap can be used to compare two different samples by mapping individual cells from a query sample either to cell-types in the reference (scmap-cluster, thick lines) or to individual cells in a reference (scmap-cell, thin lines). The comparison can be carried out either when both samples have been annotated (full lines) or when only one of them is annotated (dashed lines). (b) Cohen's $\mathrm{K}$ values and (c) percentage of unassigned cells for positive controls. The plots are based on datasets listed in Table S2 (projections are performed in both directions). Dropout-based feature selection is used for all three methods (see Methods). scmap-cell is run once for each pair of datasets. (d) Percentage of unassigned cells in negative controls. The plot is based on datasets listed in Table S3 and projections are performed in both directions. Dropout-based feature selection is used everywhere (see Methods). scmap-cell is run once for each pair of datasets. (e) scmap-cell was used to search for nearest neighbours and the accuracy shows how often the true nearest neighbour was found amongst the five or ten nearest cells. The plots are based on datasets listed in Table S1 except Shekhar and Macosko (projections are performed in both directions). Dropout-based feature selection is used (see Methods). scmap-cell is run 100 times for each dataset. 
To validate the projections, we considered 17 different datasets ${ }^{1-9,16-22}$ from mouse and human, collected and processed in different ways (Table S1). First, we evaluated different feature selection methods by carrying out a self-projection experiment where each dataset is mapped onto itself. We used $70 \%$ of the cells from the original sample for the reference and the remaining $30 \%$ were projected, with clusters as defined by the original authors. To quantify the accuracy of the mapping, we use Cohen's $\mathrm{K}^{23}$ which is a normalized index of agreement between sets of labels that accounts for the frequency of each label. A value of 1 indicates that the projection assignments were in complete agreement with the original labels, whereas 0 indicates that the projection assignment was no better than random guessing. We find that the dropout-based method for feature selection has the best performance, and somewhat surprisingly we also find that random selection is better than HVG (Fig. S1b). Furthermore, the dropout-based method performs consistently well when the number of features selected is in the range of $[100,1000]$. The dropout-based method performs better than HVG because it selects genes that are either absent or present in each cluster, and these genes provide a more reliable signal for separating groups of cells $^{14}$. As a comparison, we also considered two commonly used supervised methods for assigning labels to new samples, a random forests classifier (RF) and a support vector machine (SVM). These classifiers were trained on the reference and then applied to the held out cells as before. For the self-projection experiment, we find that both RF and SVM perform slightly better than both scmap-cluster and scmap-cell for all three feature selection methods.

As a positive control, we considered seven pairs of datasets (Table S2) that we expected to correspond well based on their origin. The positive controls represent a more realistic use-case since these comparisons include systemic differences between the reference and projection samples, i.e. batch effects. For example, for three of the pairs, one of the datasets was collected using a full-length protocol and the other was collected using a UMI based protocol. The results showed that despite the substantial differences between the protocols ${ }^{16,24,25}$, both scmap-cluster and scmap-cell on average achieve $\mathrm{k}>.75$ and assignment rates $>75 \%$ when the number of features used was between 100 and 500 (Fig. 1b, c, S2a, S3). Even though RF achieves the highest $\mathrm{K}$ of the three approaches it also has the lowest assignment rate $(<50 \%)$ indicating that it achieves a high specificity at the cost of low sensitivity. An important feature of scmap is that it is robust to gene dropouts since both the centroids and the nearest neighbour relations are unaffected by the increased frequency of zeros (Fig. S4).

As a negative control, we projected datasets with an altogether different origin from the reference (e.g. mouse retina onto mouse pancreas, Table S3). Reassuringly, we found that both scmap versions categorized $>90 \%$ of the cells as unassigned when the number of features used was $>100$ (Fig. 1d). Notably, SVM has a much smaller fraction of unassigned cells than RF and scmap, indicating that it is too lenient in assigning matches. Comparing the evidence across the self-projection experiments, the positive and negative controls, we conclude that scmap with 500 features provides the best performance by balancing high sensitivity and specificity with a low false-positive rate.

We evaluated the scmap-cell by asking how often it was able to identify the true nearest neighbor, as defined by calculating the nearest neighbor exactly, amongst one of the five or ten nearest cells. For 15 of the 17 datasets used earlier, scmap has an average accuracy of $64 \%$ or $80 \%$, 
respectively (Fig. 1e). For the two Drop-seq datasets, scmap-cell performed well in identifying the correct cluster, yet it only achieved an accuracy of $\sim 20 \%$ for identifying the nearest neighbor. We hypothesize that deeper sequencing is required for scmap-cell to be able to reliably identify nearest neighbors. The ANN search is most useful for differentiation trajectories where the cells are typically thought to best be represented as a continuum rather than discrete clusters ${ }^{26}$. We evaluated the ANN feature of scmap for trajectories for mouse myoblast differentiation ${ }^{27}$, mouse ES differentiation ${ }^{28}$ and mouse fibroblast to neuron reprogramming ${ }^{29}$. We again found that scmap can correctly identify the nearest neighbor in $76 \%, 91 \%$, and $94 \%$ of the cases (Fig. 2a).

An important feature of scmap is that it is very fast. It takes only around twenty seconds to select features and to calculate the centroids for 40,000 cells for scmap-cluster, for scmap-cell it takes less than one minute to create the index, whereas it takes almost thirty minutes to train using RF or SVM (Fig. 2b). For all four methods the time to project the new cells is negligible, which means they are very fast with a pre-computed reference. Since the complexity scales with the number of clusters in the reference, rather than the number of cells, scmap-cluster will be applicable to very large datasets as the index is $\sim 5000$ times smaller than the original expression matrix. The scmap-cell index is $\sim 500$-fold smaller than the original expression matrix (Table S1).

Large references, including the HCA, will be an agglomeration of datasets collected by different groups. Merging different scRNA-seq datasets remains an open problem ${ }^{30-32}$, but the results from our study suggest that samples with similar origin are largely consistent ${ }^{33}$ (Fig. 1c). Instead of correcting for batches and merging, one can create a composite reference and compare the new cells to each dataset separately. When there are multiple datasets in the reference, scmap reports the best match for each dataset. Thus, if a cell shows a high degree of similarity to clusters with similar annotations from different datasets, that will increase the confidence of the mapping. To illustrate the mapping to multiple datasets, we considered the pancreas dataset by Baron et al ${ }^{4}$ since it had the most unassigned cells when projected to the other pancreas datasets. Combining all projections (Methods) we were able to reduce the fraction of unassigned cells from $99 \%\left(\mathrm{Xin}^{3}\right.$ ) and $88 \%$ (Segerstolpe ${ }^{1}$ ) to $63 \%$ while not making $\mathrm{k}$ worse. Interestingly, for this example the performance of scmap-cell was better than scmap-cluster (Fig. 2c-e). Since the reference used by scmap is modular and can be extended without re-calculating the features or centroids for the datasets that have been processed previously, the strategy of not merging datasets is well suited for large references that are expected to grow over a long period of time. 

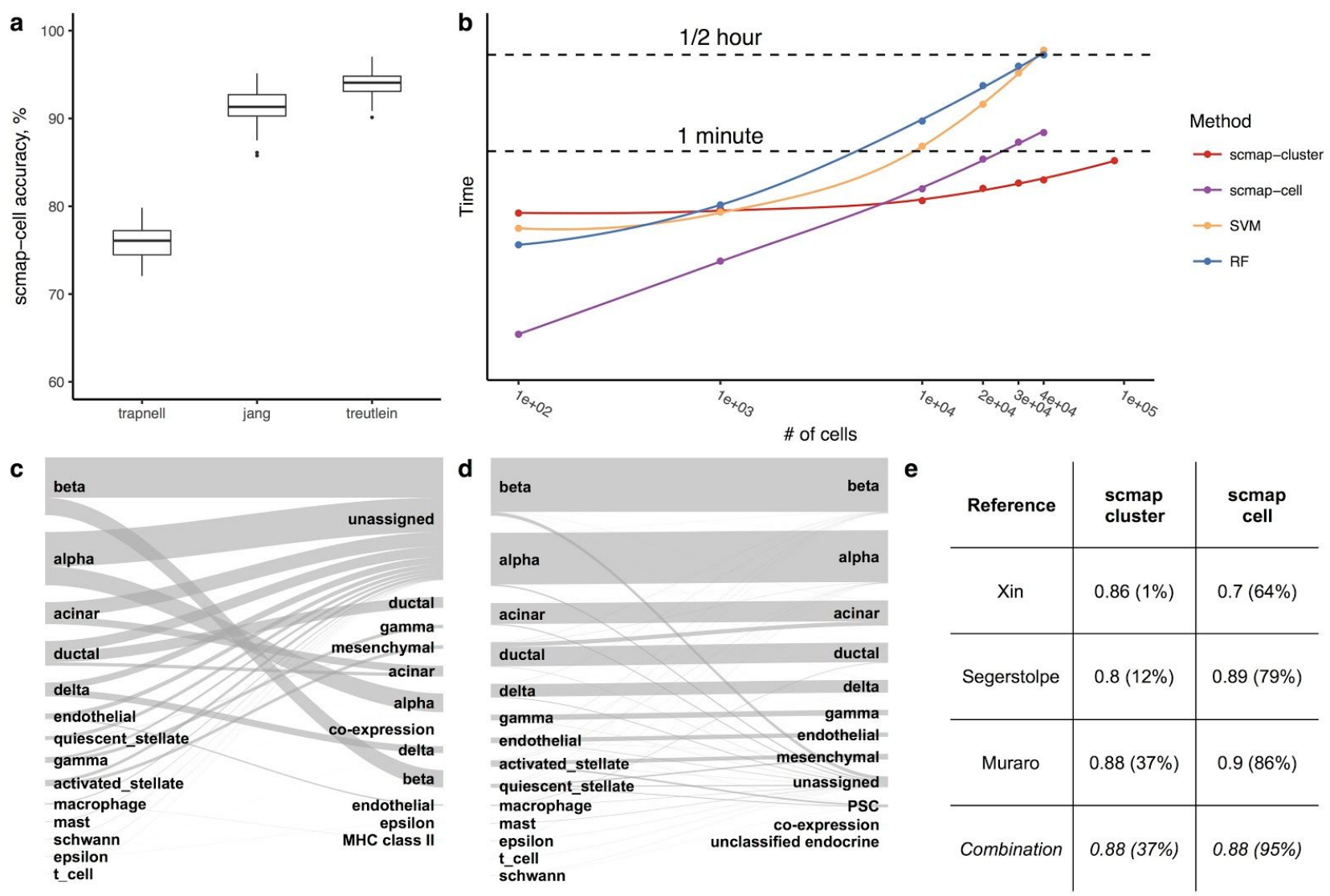

Figure 2. scmap for combined references. (a) scmap-cell accuracy for three datasets ${ }^{27-29}$ with differentiation trajectories showing how often the true nearest neighbour was found amongst the ten nearest cells (1000 dropout-selected features were used for projections and scmap-cell was run 100 times for each dataset). (b) CPU run times of creating Reference (for scmap) and training classifiers (for SVM and RF). The $x$-axis represents a number of cells in the reference dataset. For all methods 1,000 features were used. All methods were run on a MacBook Pro laptop (Mid 2014) with $2.8 \mathrm{GHz}$ Intel Core i7 processor, 16 GB 1600 $\mathrm{MHz}$ DDR3 of RAM. For $10^{5}$ cells, scmap-cell failed due to lack of memory. Points are actual data, solid lines are "loess" fit to the points with span $=1$ (see ggplot2 documentation). (c) Results of scmap-cluster projection of the Baron ${ }^{4}$ dataset to Muraro ${ }^{2}$, Segerstolpe ${ }^{1}$ and $\mathrm{Xin}^{3}$ dataset using a combination strategy (Sankey diagram) and (d) scmap-cell projection (Sankey diagram). (e) Results of scmap-cluster and scmap-cell projections of the Baron ${ }^{4}$ dataset to three human pancreas datasets (Reference) and results of the Combination projection.

We have implemented scmap as an R-package, and it is part of Bioconductor to facilitate incorporation into bioinformatic workflows. Since scmap is integrated with scater ${ }^{34}$, it is easy to combine with many other popular computational scRNA-seq methods. Moreover, we have made scmap available via the web (http://www.hemberg-lab.cloud/scmap), allowing users to either upload their own reference, or to use a reference collection of datasets from this paper for which the features and centroids have been pre-calculated (Methods).

Due to differences in experimental conditions, comparing scRNA-seq datasets remains challenging. However, for researchers to be able to take advantage of large references, e.g. the HCA, fast, robust and accurate methods for merging ${ }^{35,36}$ and projecting cells across datasets are required. To the best of our knowledge, scmap is the first widely applicable projection method since it can identify both the best matching cell-type as well as individual cell in the reference. We 
have demonstrated that scmap can be used to compare samples of similar origin collected by different groups, as well as for comparing cells to a large reference composed of multiple datasets.

\section{Methods}

\section{Datasets}

All datasets and cell type annotations were downloaded from their public accessions. The datasets were converted into Bioconductor's SingleCellExperiment (http://bioconductor.org/packages/SingleCellExperiment) class objects (details are available on our dataset website: https://hemberg-lab.github.io/scRNA.seq.datasets). In the Segerstolpe ${ }^{1}$ dataset cells labeled as "not applicable" were removed since it is unclear how to interpret this label and what it should be matched to in the other datasets. In the $\mathrm{Xin}^{3}$ dataset cells labeled as "alpha.contaminated", "beta.contaminated", "gamma.contaminated" and "delta.contaminated" were removed since they likely correspond to cells of lower quality. In the following datasets similar cell types were merged together:

- In the Deng ${ }^{17}$ dataset zygote and early2cell were merged into zygote cell type, mid2cell and late2cell were merged into 2cell cell type, and earlyblast, midblast and lateblast were merged into blast cell type.

- All bipolar cell types of the Shekhar ${ }^{9}$ dataset were merged into bipolar cell type.

- In the Yan ${ }^{21}$ dataset oocyte and zygote cell types were merged into zygote cell type.

\section{Feature selection}

To select informative features we used a method conceptually similar to M3Drop ${ }^{14}$ to relate the mean expression $(E)$ and the dropout rate $(D)$. We used a linear model to capture the relationship $\log (E)$ and $\log (D)$, and after fitting a linear model using the $\operatorname{lm}()$ command in $\mathrm{R}$, important features were selected as the top $N$ residuals of the linear model (Fig. S1a). The features are only selected from the reference dataset, and those of them absent or zero in the projection dataset are are further excluded before running scmap. All three feature selection methods are described in Supplementary Note 1.

\section{Reference centroid}

In scmap-cluster each cell type in the reference dataset is represented by its centroid, i.e. the median value of gene expression for each feature gene across all cells in that cell type.

\section{Approximate nearest neighbor search using product quantizer}

scmap-cell performs fast approximate k-nearest neighbour search using product quantization ${ }^{13}$. The original algorithm, built around the Euclidean distance, was adapted to incorporate the cosine distance, which helps to protect against batch effects and scaling inconsistencies between 
datasets. The product quantizer creates a compressed index where every cell in the reference is identified with a set of sub-centroids found via k-means clustering based on a subset of the features. By concatenating the sub-centroids, a close approximation to the original expression vector is obtained. When searching the reference for the nearest neighbours to a query cell, the approximations provided by the sub-centroids are used instead of the individual cells in the reference. Since the number of centroids can be made much smaller than the original number of cells in the dataset, the method provides a substantial reduction in both computation time and storage requirements compared to exact search.

\section{Projection dataset}

Projection of a dataset to a reference dataset is performed by calculating similarities between each cell and all centroids of the reference dataset, using only the common selected features. Three similarity measures are used: Pearson, Spearman and cosine. The cell is then assigned to the cell type which correspond to the highest similarity value. However, scmap-cluster requires that at least two similarity measures agree with each other, otherwise the cell is marked as "unassigned". Additionally, if the maximum similarity value across all three similarities is below a similarity threshold (default is .7), then the cell is also marked as "unassigned". Since only the cosine similarity measure was calculated for scmap-cell, the default threshold of .5 was used, and the nearest three neighbours are required to agree on the cell-type for it to be assigned. Positive and negative control plots corresponding to Figs. 1c-e for different values of the similarity/probability (see SVM and RF) threshold (.5, .6, .8 and .9) are shown in Fig. S2.

\section{SVM and RF}

The scmap projection algorithm was benchmarked against support vector machines ${ }^{37}$ (with a linear kernel) and random forests ${ }^{38}$ (with 50 trees) classifiers from the $R$ packages $\mathrm{e} 1071$ and randomForest. The classifiers were trained on all cells of the reference dataset and a cell type of each cell in the projection dataset was predicted by the classifiers. Additionally, a threshold (default value of .7) was applied on the probabilities of assignment: if the probability was less than the threshold the cell was marked as "unassigned".

\section{Sensitivity to sequencing depth and dropouts}

The dropout rate in the positive control datasets (Table S2) was artificially increased by randomly setting $10 \%, 30 \%$ and $50 \%$ of the non-zero expression values to zero (Fig. S4a,b). scmap was run 100 times for each box.

\section{Projection based on multiple datasets}

When the reference contains multiple datasets collected from similar samples by different groups in addition to all similarities, for each cell scmap also reports a top cell type match based on the highest value of similarities across all reference cell types. A similarity threshold of .7 is also applied in this case. 
bioRxiv preprint doi: https://doi.org/10.1101/150292; this version posted November 29, 2017. The copyright holder for this preprint (which was not certified by peer review) is the author/funder, who has granted bioRxiv a license to display the preprint in perpetuity. It is made available under aCC-BY-NC 4.0 International license.

An example of a cloud version of scmap is available at http://www.hemberg-lab.cloud/scmap. Instructions of how to set it up on a user's personal web cloud environment are available on our github page: https://github.com/hemberg-lab/scmap-shiny. An extended tutorial on how to use scmap can be found in Supplementary Note 2.

\section{Figures}

All data and scripts used to generate figures in this paper are available at https://github.com/hemberg-lab/scmap-paper-figures. 


\section{Acknowledgements}

We would like to thank Tallulah Andrews, Kedar N Natarajan, Guillermo Parada, Michael Schaub, Michael Stubbington, Valentine Svensson, Jennifer Westoby and Florian Wünnermann for helpful discussions, feedback on the manuscript and for testing the cloud implementation of scmap.

\section{Funding}

Amazon Web Services (AWS) Cloud provided credits for running the scmap server for one year. VYK and MH were both supported by core funding to the Wellcome Trust Sanger Institute provided by the Wellcome Trust.

\section{Conflicts of interest}

None.

\section{Author contributions}

M.H. conceived the study and supervised the research; V.Y.K., A.Y. and M.H. contributed to the computational framework; V.Y.K. and M.H. wrote the manuscript. 


\section{References}

1. Segerstolpe, A. et al. Single-Cell Transcriptome Profiling of Human Pancreatic Islets in Health and Type 2 Diabetes. Cell Metab. 24, 593-607 (2016).

2. Muraro, M. J. et al. A Single-Cell Transcriptome Atlas of the Human Pancreas. Cell Syst 3, 385-394.e3 (2016).

3. Xin, Y. et al. RNA Sequencing of Single Human Islet Cells Reveals Type 2 Diabetes Genes. Cell Metab. 24, 608-615 (2016).

4. Baron, M. et al. A Single-Cell Transcriptomic Map of the Human and Mouse Pancreas Reveals Inter- and Intra-cell Population Structure. Cell Syst 3, 346-360.e4 (2016).

5. Zeisel, A. et al. Brain structure. Cell types in the mouse cortex and hippocampus revealed by single-cell RNA-seq. Science 347, 1138-1142 (2015).

6. Tasic, B. et al. Adult mouse cortical cell taxonomy revealed by single cell transcriptomics. Nat. Neurosci. 19, 335-346 (2016).

7. Usoskin, D. et al. Unbiased classification of sensory neuron types by large-scale single-cell RNA sequencing. Nat. Neurosci. 18, 145-153 (2015).

8. Macosko, E. Z. et al. Highly Parallel Genome-wide Expression Profiling of Individual Cells Using Nanoliter Droplets. Cell 161, 1202-1214 (2015).

9. Shekhar, K. et al. Comprehensive Classification of Retinal Bipolar Neurons by Single-Cell Transcriptomics. Cell 166, 1308-1323.e30 (2016).

10. Kiselev, V. Y. et al. SC3: consensus clustering of single-cell RNA-seq data. Nat. Methods (2017). doi:10.1038/nmeth.4236

11. Regev, A. et al. The Human Cell Atlas. bioRxiv 121202 (2017). doi:10.1101/121202

12. Altschul, S. F., Gish, W., Miller, W., Myers, E. W. \& Lipman, D. J. Basic local alignment search tool. J. Mol. Biol. 215, 403-410 (1990).

13. Jégou, H., Douze, M. \& Schmid, C. Product quantization for nearest neighbor search. IEEE Trans. Pattern Anal. Mach. Intell. 33, 117-128 (2011). 
bioRxiv preprint doi: https://doi.org/10.1101/150292; this version posted November 29, 2017. The copyright holder for this preprint (which was not certified by peer review) is the author/funder, who has granted bioRxiv a license to display the preprint in perpetuity. It is made available under aCC-BY-NC 4.0 International license.

14. Andrews, T. S. \& Hemberg, M. Modelling dropouts for feature selection in scRNASeq experiments. bioRxiv 065094 (2017). doi:10.1101/065094

15. Brennecke, P. et al. Accounting for technical noise in single-cell RNA-seq experiments. Nat. Methods 10, 1093-1095 (2013).

16. Klein, A. M. et al. Droplet Barcoding for Single-Cell Transcriptomics Applied to Embryonic Stem Cells. Cell 161, 1187-1201 (2015).

17. Deng, Q., Ramsköld, D., Reinius, B. \& Sandberg, R. Single-cell RNA-seq reveals dynamic, random monoallelic gene expression in mammalian cells. Science 343, 193-196 (2014).

18. Goolam, M. et al. Heterogeneity in Oct4 and Sox2 Targets Biases Cell Fate in 4-Cell Mouse Embryos. Cell 165, 61-74 (2016).

19. Pollen, A. A. et al. Low-coverage single-cell mRNA sequencing reveals cellular heterogeneity and activated signaling pathways in developing cerebral cortex. Nat. Biotechnol. 32, 1053-1058 (2014).

20. Li, H. et al. Reference component analysis of single-cell transcriptomes elucidates cellular heterogeneity in human colorectal tumors. Nat. Genet. 49, 708-718 (2017).

21. Yan, L. et al. Single-cell RNA-Seq profiling of human preimplantation embryos and embryonic stem cells. Nat. Struct. Mol. Biol. 20, 1131-1139 (2013).

22. Kolodziejczyk, A. A. et al. Single Cell RNA-Sequencing of Pluripotent States Unlocks Modular Transcriptional Variation. Cell Stem Cell 17, 471-485 (2015).

23. Cohen, J. Weighted kappa: nominal scale agreement with provision for scaled disagreement or partial credit. Psychol. Bull. 70, 213-220 (1968).

24. Hashimshony, T. et al. CEL-Seq2: sensitive highly-multiplexed single-cell RNA-Seq. Genome Biol. 17, 77 (2016).

25. Picelli, S. et al. Full-length RNA-seq from single cells using Smart-seq2. Nat. Protoc. 9, $171-181(2014)$

26. Wagner, A., Regev, A. \& Yosef, N. Revealing the vectors of cellular identity with single-cell 
genomics. Nat. Biotechnol. 34, 1145-1160 (2016).

27. Trapnell, C. et al. The dynamics and regulators of cell fate decisions are revealed by pseudotemporal ordering of single cells. Nat. Biotechnol. 32, 381-386 (2014).

28. Jang, S. et al. Dynamics of embryonic stem cell differentiation inferred from single-cell transcriptomics show a series of transitions through discrete cell states. Elife 6, (2017).

29. Treutlein, B. et al. Dissecting direct reprogramming from fibroblast to neuron using single-cell RNA-seq. Nature 534, 391-395 (2016).

30. Camp, J. G. et al. Multilineage communication regulates human liver bud development from pluripotency. Nature 546, 533-538 (2017).

31. Tung, P.-Y. et al. Batch effects and the effective design of single-cell gene expression studies. Sci. Rep. 7, 39921 (2017).

32. La Manno, G. et al. Molecular Diversity of Midbrain Development in Mouse, Human, and Stem Cells. Cell 167, 566-580.e19 (2016).

33. Crow, M., Paul, A., Ballouz, S., Josh Huang, Z. \& Gillis, J. Addressing the looming identity crisis in single cell RNA-seq. bioRxiv 150524 (2017). doi:10.1101/150524

34. McCarthy, D. J., Campbell, K. R., Lun, A. T. L. \& Wills, Q. F. Scater: pre-processing, quality control, normalization and visualization of single-cell RNA-seq data in R. Bioinformatics (2017). doi:10.1093/bioinformatics/btw777

35. Haghverdi, L., Lun, A. T. L., Morgan, M. D. \& Marioni, J. C. Correcting batch effects in single-cell RNA sequencing data by matching mutual nearest neighbours. bioRxiv 165118 (2017). doi:10.1101/165118

36. Butler, A. \& Satija, R. Integrated analysis of single cell transcriptomic data across conditions, technologies, and species. bioRxiv 164889 (2017). doi:10.1101/164889

37. Ben-Hur, A., Horn, D., Siegelmann, H. T. \& Vapnik, V. Support Vector Clustering. J. Mach. Learn. Res. 2, 125-137 (2002).

38. Breiman, L. Random Forests. Mach. Learn. 45, 5-32 (2001). 
bioRxiv preprint doi: https://doi.org/10.1101/150292; this version posted November 29, 2017. The copyright holder for this preprint (which was not certified by peer review) is the author/funder, who has granted bioRxiv a license to display the preprint in perpetuity. It is made available under aCC-BY-NC 4.0 International license. 